\title{
An Experiment in Parallelizing an Application Using Formal Methods
}

\author{
Computer Aided Parallelization
}

\author{
Raphaël Couturier and Dominique Méry \\ UMR $n^{\circ} 7503$ LORIA CNRS \\ Université Henri Poincaré \\ BP 239, 54506 Vandœuvre-lès-Nancy \\ France \\ email: couturie,mery@loria.fr
}

\begin{abstract}
Scientists and engineers have successfully developed much useful sequential code using classical programming languages, such as Fortran, and highly performant computers can help them to model problems whilst improving their results of simulation. However, great skill and care are required to design correct parallel code from sequential code. We focus our work on 3 aspects of producing correct parallel code from sequential code. First, we use a theorem prover, namely PVS, as a practical tool for asserting proof obligations and for stating programs and properties. Secondly, we build an abstraction from some sequential code in order to derive a postcondition. Finally, we characterize the "glueing condition" that will ensure the correctness of the final parallel code. Our method does not need any new notation but reuses proof theoretical notations together with the composition of postconditions with respect to the glueing condition.
\end{abstract}

\section{Introduction}

Parallel program design is a very challenging topic that requires formal techniques for guaranteeing both safety and liveness properties; furthermore, parallel programming poses questions with regards to the efficiency of the designed programs and on the relationship of the designed program to a given architecture. The design of parallel programs has been greatly influenced by the seminal framework of Chandy and Misra, namely UNITY [2], where a new perspective was suggested: a general framework for stating programs, properties and mapping shows how formal techniques could be put together in a uniform notation. The concept of refinement is a very crucial point in the UNITY philosophy: $a$ "text" refines another "text" when what is true for the first "text" is also true for the second text. The formal interpretation of this statement is founded on the notion of predicate transformers $[4,5]$ and the choice of a language for specifying properties such as invariance or eventuality. Our paper tackles the question of parallelization of sequential code provided by non-computer scientists ${ }^{1}$, under

\footnotetext{
1 Physics, Chemistry
} 
extra assumptions for preserving semantics through transformations by analyzing the code with a theorem prover, namely PVS [10], and by reasoning with a formal notation integrating proof methods. Moreover, our case study, has produced parallel executable code for a high peformance parallel computer ${ }^{2}$, in our laboratory, which is actually used by the original scientists.

Before stating the method we have used in the parallelization of a Monte Carlo simulation of a grid of spins, we review different critical points related to the use of a formal method. We are not proposing an implementation of the UNITY notation in PVS but we do use the UNITY philosophy to separate a formal reasoning into an intermediate notation, namely PVS, together with the implementation in a parallel programming language (a parallel version of Fortran). We want to avoid the myth of the universal theorem prover and try to convince our non-computer scientists colleagues that tools may improve their work and help them to build parallel programs. The underlying model for stating algorithmic notations is based on UNITY but can also be related to TLA [8], VDM [6] and $Z$ [13], where actions are also expressed using post-states with primed notation. We are interested by properties stating that when the program halts a postcondition holds, and this requires the use of invariants and the generation of proof obligations. The main problem is to derive a global property from local properties of pieces of the global program when it is parallelized. We limit our study to programs that are supposed to terminate in the manner of Chandy and Taylor [3]. Our problem is to organize the computations to preserve the total correctness of the initial program.

Our method consists of abstracting from an existing sequential code using the specification language of a prover, namely PVS [10]. The specification must explicily state the postconditions of the parallel parts. Our aim is to use postconditions and to prove, with the help of a prover, that postconditions obtained with the parallel program imply postconditions resulting from the sequential program, under an extra glueing condition, which ensures that pieces of programs cooperate properly. This condition is either given by the user or stated by the system. We apply this method to a Monte Carlo simulation of a grid of spins [11]. Our problem has a large number of iterations (about one million) and uses a huge number of random numbers (about $10^{14}$ ). During each iteration, there are four steps. Three steps are easy to parallelize, but the fourth requires more attention because it involves the creation of graphs which may be on the whole grid. It is difficult to argue without a formal approach that the transformed code is correct with respect to the initial specification.

The paper is organized as follows. Section two describes our method. Section three explains the Monte Carlo simulation. Section four parallelizes the application. Section five proves, with the help of PVS, that our parallelization concerning the graphs is correct. Section six explains how a prover can be used to discover postconditions guaranteeing a correct program parallelization, and to state the glueing condition. Section seven presents other work related to our problem. Section eight concludes our paper.

${ }^{2}$ The Silicon Graphics Origin 2000 of the Charles Hermite Center 


\section{Using postconditions and a prover to parallelize an algorithm}

The proof of a parallel program is a hard task, which can be done in at least two ways. On the one hand, we can develop the program and then prove it. Or, on the other hand, we can specify it, prove it and then develop it using the specification. The technique we are proposing can be used with both these methods.

In general when we parallelize a program, we already have the sequential version and the parallel code is founded on this. With more or less abstraction, the parallel parts of the code are fragments of the sequential code. Thus, the postcondition of a sequential code part is the same as the equivalent part in the parallel code, if this postcondition is well defined.

Next, given suitably defined postconditions, we must define the postcondition of the glue code which assembles all parts computed in parallel. If all these postconditions are well defined with a good level of abstraction ${ }^{3}$, it is possible to prove that the postconditions of parallel code plus the postconditions of the glueing code implies the postconditions of the equivalent sequential code. This first method is schematized in Fig1(a).

The alternative method consists of first realizing an abstraction of the sequential algorithm and thus specifying the postconditions of the algorithm. Then, when we try to prove that postconditions of the parallel code without any glue conditions, all the proof obligations may not be proven. This is normal because the postcondition of the glue code is not specified. However, all the failed proof obligations are the required postconditions of the glue code. This second method is schematized in Fig1(b).

\section{The Monte Carlo simulation}

In this section, we explain briefly the simulation of Monte Carlo we use to validate our method, and describe the problems this parallelization implies. We take sequential code, developed in the Laboratoire de physique des Matériaux, by B. Berche, P.E. Berche and C. Chatelain [11]. This application is based on a large number of interdependent iterations, but each iteration is not time consuming (less than one second). We dispose spins on nodes of a rectangular net. The initial net was a grid $100^{*} 100$. These spins follow a Markov law, that is to say that their evolution is based on a given probabilistic transition of a current configuration to the next one. At each iteration, thousands or millions of random numbers are generated (depending on the size of the given problem).

Now we present the 4 different steps involved in each iteration. The first step consists of randomly destroying links of the grid with a given probability. Next, the second step consists of exploring the grid to create graphs using existing

\footnotetext{
${ }^{3}$ If the level of abstraction is too high, the proof would be very difficult; and, if it is too low, the postcondition would not be useful.
} 


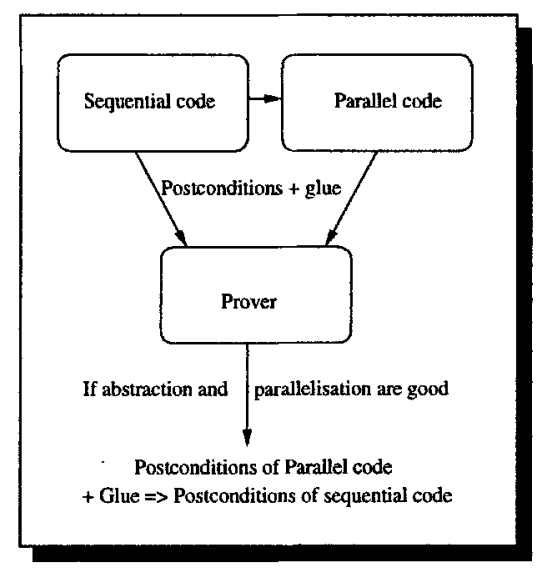

(a) proof that parallelization is correct with help of a prover

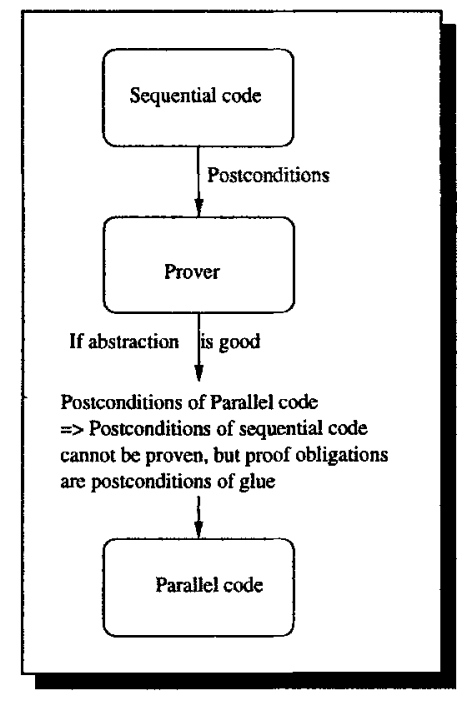

(b) parallelization with help of a prover

Fig. 1. The two methods we are proposing

links (there are either horizontal links or vertical links). Then links must be updated using graphs obtained during step two. Finally, we must calculate physical measures modeled by the system.

One of the most difficult problems to parallelize with this application is that we always want to generate the same random numbers with the parallel code as with the sequential code; but the random number generator, namely RANDAR [15], used for its properties (weak correlation...), cannot be parallelized, except by making time consuming concessions, because it is a combination of two generators. We overcome this constraint by generating the same random numbers for the initialization of the simulation process and then using different generators with different seeds.

Using this approach, we obtain converging results using sequential and parallel simulation after several iterations.

\section{Parallelization of the simulation}

In this section we present the parallelization which was made on the Silicon Graphics Origin 2000 at the Charles Hermite Center, LORIA, in Nancy. This machine possesses 64 processors with a distributed shared memory. The initial code is written in Fortran 77, so we keep this language and we use its parallel version on the Origin. Steps 1, 3 and 4 can be parallelized quite easily using band 
or grid decomposition, since there is no interaction between different points of the grid. We should note that in some cases, corresponding to small computations, the parallelization does not give any acceleration. To parallelize the code, we add compiler directives before DO loops and we specify, for each variable involved in a loop, if it is a shared, local or reduction variable. In this way, each iteration in which there are no dependance between variables can be executed in parallel. Thus the number of iterations is dispatched dynamicaly between all the threads created by the program. This method has similitudes with the data-parallelism. All the work concerning the creation, synchronisation or destruction of threads is made by the compiler. The use of directives is advantageous compared to the traditional way of parallelizing code (PVM [14], MPI [12]) because they can be ignored and the code can be compiled to obtain a sequential implementation. This reduces the time required to develop the parallel code.

Whereas computation was easy to parallelize in steps 1,3 and 4, step 2 is more complex. In fact, the graph problem cannot be divided without precaution since we have no information concerning graph locations, and hence they can be located anywhere on the grid. To complete this step, we must build all connected components of the grid. Information to build these components belongs to 2 arrays which may be either in shared memory (in our case) or distributed on local memory (in a distributed system). Thus, to solve this graph problem consuming as little time as possible, we split the initial grid into sub-grids. Then, using the same algorithm as in the sequential case, we can build partial graphs on sub-grids in parallel. When all the partial graphs are built, an assembly step (we call this the glue step), is required in order to obtain the same graphs as would be seen in the sequential computation ( this last step is realized sequentially because we do not want to use critical sections, which are time consuming in our case). The figure Fig2 shows how an example of two partial graphs which are glued and the resulting graph.

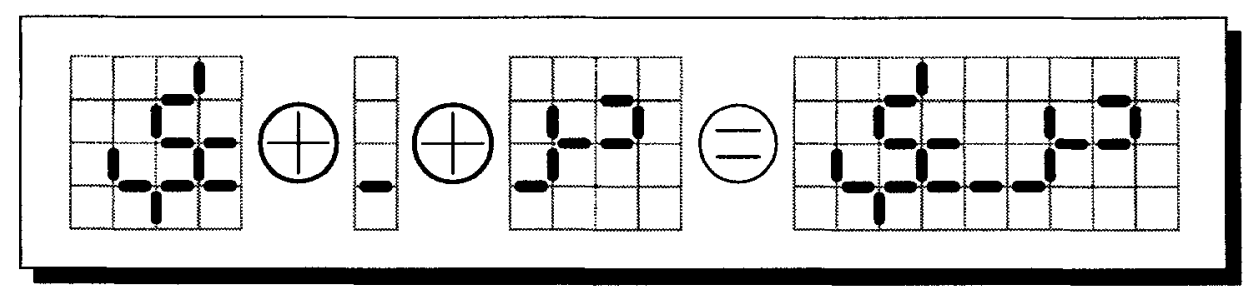

Fig. 2. Glueing of two graphs obtained in sequential

Previously, we stated that the random number generator we used could not be parallelized even though the generation of random numbers is a crucial task in this simulation. Thus the substitute method used, consisting of generating the same initial numbers with the same generator and then using different generators with different seeds, must provide, at the end of the simulation, a result very 
close to the sequential one. This property can be observed in our case, but some applications may exist which would require another method to parallelize the random number generator. This was beyond the scope of this paper.

The performance gains we obtain are given below. The time is expressed in seconds.

Here is the resume of the used steps:

step 1: destruction of links

step 2: graphs construction

step 3: links updates

step 4: measures

- 100000 iterations on a $200^{*} 200$ grid

\begin{tabular}{|c|c|c|c|c|c|c|}
\hline nb proc & step 1 & step 2 & step 3 & step 4 & an iteration & speed up \\
\hline 1 & 3397 & 3581 & 767 & 432 & 0.082 & 1 \\
\hline 4 & 892 & 1051 & 234 & 536 & 0.027 & 3 \\
\hline
\end{tabular}

- 10000 iterations on a $600^{*} 600$ grid

\begin{tabular}{|c|c|c|c|c|c|c|}
\hline $\mathrm{nb}$ proc & step 1 & step 2 & step 3 & step 4 & an iteration & speed up \\
\hline 1 & 3046 & 3323 & 686 & 536 & 0.76 & 1 \\
\hline 4 & 965 & 877 & 223 & 745 & 0.28 & 2.7 \\
\hline 9 & 423 & 651 & 94 & 281 & 0.14 & 5.4 \\
\hline 16 & 230 & 598 & 57 & 117 & 0.10 & 7.6 \\
\hline
\end{tabular}

Despite the overload of work, it is amazing to see that the graphs creation step is fast. We must say that only the fact of splitting the grid allows a performance gain even in the sequential case (due to the cache memory of the machine). The overal speedup is between 2.7 and 3 with 4 processors. With more processors, the efficiency gains are less attractive. This relatively bad performance with more processors is explained by the use of frequent synchronisations inherent in the programming model. The Fortran compiler synchronises all the threads or processes after each DO loop and, in the present computation, there are several short loops.

\section{Proof that the "graph parallelization" is correct}

In this section we prove, with the help of the PVS prover, that the parallelization of the graphs creation step is correct. For this we use the method described in Fig1(a). We consider the case where we have only 4 processors and so we decompose the initial grid into 4 sub-grids. We start by establishing the postcondition of the sequential code concerned with this step.

In the Fortran code, we use 2 arrays, HLink and VLink, containing horizontal and vertical links for each point of the grid. After the graphs creation step, results are returned in another array called Spin. 
Using the formalism of PVS [10], we define 2 types for arrays of nat and bool. The variable Spinp is the array containing all the graphs in the sequential case, whereas Spin2p contains all graphs in the parallel case (we use the letter $p$ in reference to the concept of primed variable in TLA [8]).

Array2_nat : TYPE $=[$ nat, nat $->$ nat $]$

Array2_bool : TYPE $=[$ nat, nat $->$ bool $]$

Spinp,Spin2p : Var Array2_nat

HLink, VLink : Var Array2_bool

Now, we define the postcondition obtained after the execution of the sequential algorithm. As we use the same algorithm in the parallel code, this postcondition is also verified after each computation on the sub-grids. The variables of $f x$ and off_y represent, respectively, an $x$ and an $y$ offset of the grid from which we apply the algorithm. We use offsets to be able to apply the same algorithm at different locations on the grid, and so parallelize this step.

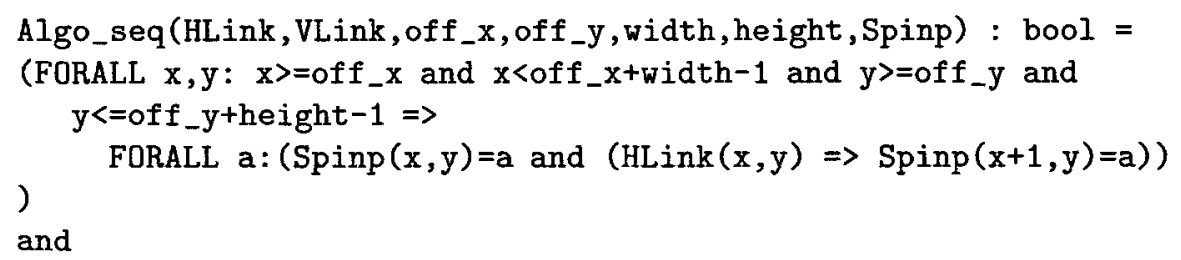

In this formula, we specify that for all well defined $\mathrm{x}$ and $\mathrm{y}$, if after the execution of the algorithm we obtain a value $a$ in $\operatorname{Spinp}(x, y)$ and if there is a HLink in $(x, y)$, then we have $\operatorname{Spinp}(x+1, y)=a$. We have a similar property with VLink. We quantify a because we do not know in advance the value of this variable.

Then we specify the glue condition. The Verticalglueing for instance, says that for $x$ and $y$ belonging to the boundary, if $\operatorname{Spin} 2 p(x, y)$ contains a value in the right grid and if $\operatorname{Spin} 2 \mathrm{p}(\mathrm{x}+1, y)$ contains another value, then if there is a HLink in $(x, y)$ then this implies that both the values are equal.

VerticalGlueing (HLink, VLink, off_x, width, height,Spin2p): bool = FORALL $x, y: \quad\left(x=o f f_{-} x\right.$ and $y>=0$ and $y<$ height-1 $\Rightarrow$

FORALL $a, b:(\operatorname{Spin} 2 p(x, y)=a$ and $\operatorname{Spin} 2 p(x+1, y)=b) \Rightarrow$

$\operatorname{HLink}(x, y) \Rightarrow a=b)$

HorizontalGlueing (HLink, VLink, off_y, width, height, Spin2p) : bool = FORALL $x, y$ : ( $y=0 f_{-}-y$ and $x>=0$ and $x<$ width-1 $\Rightarrow$

FORALL $a, b:(\operatorname{Spin} 2 p(x, y)=a$ and $\operatorname{Spin} 2 p(x, y+1)=b) \Rightarrow$

$\operatorname{VLink}(x, y) \Rightarrow a=b)$ 
Now, we just have to specify what exactly we want to prove. We have the specification of the sequential algorithm (which is the same as the parallel one), and we have the glue postconditions. As we use only 4 sub-grids (for this proof), we must prove that:

EQUIVALENCE : THEOREM FORALL $\mathrm{k} 1, \mathrm{k} 2$ : $\mathrm{k} 1=$ width/2 and $\mathrm{k} 2=$ height $/ 2$ and width $>=2$ and height $>=2$ => (

(

Algo_seq(HLink,VLink, 0,0,width, height,Spinp) and Algo_seq(HLink, VLink, $0,0, \mathrm{k} 1, \mathrm{k} 2$, Spin2p) and Algo_seq(HLink, VLink, k1,0,k1,k2,Spin2p) and Algo_seq(HLink,VLink, 0,k2,k1,k2,Spin2p) and Algo_seq(HLink, VLink, k1,k2,k1,k2,Spin2p) and VerticalGlueing (HLink,VLink,k1-1,width, height,Spin2p) and HorizontalGlueing (HLink, VLink, k2-1, width, height, Spin2p) )

$\Rightarrow$

(

(FORALL $\mathrm{x}, \mathrm{y}: \mathrm{x}>=0$ and $\mathrm{y}>=0$ and $\mathrm{x}<$ width -1 and $\mathrm{y}<$ height $-1 \Rightarrow$ (FORALL $a, b, c:$

(

$(\operatorname{Spinp}(x, y)=a$ and $\operatorname{Spin} 2 p(x, y)=b$ and $\operatorname{Spin} 2 p(x+1, y)=c) \Rightarrow$ $(\operatorname{HLink}(x, y) \Rightarrow(\operatorname{Spinp}(x+1, y)=a$ and $\operatorname{Spin} 2 p(x+1, y)=b))$ )

)

) and

(FORALL $\mathrm{x}, \mathrm{y}: \mathrm{x}>=0$ and $\mathrm{y}>=0$ and $\mathrm{x}<$ width-1 and $\mathrm{y}<$ height $-1 \Rightarrow$ (FORALL $a, b, c$ :

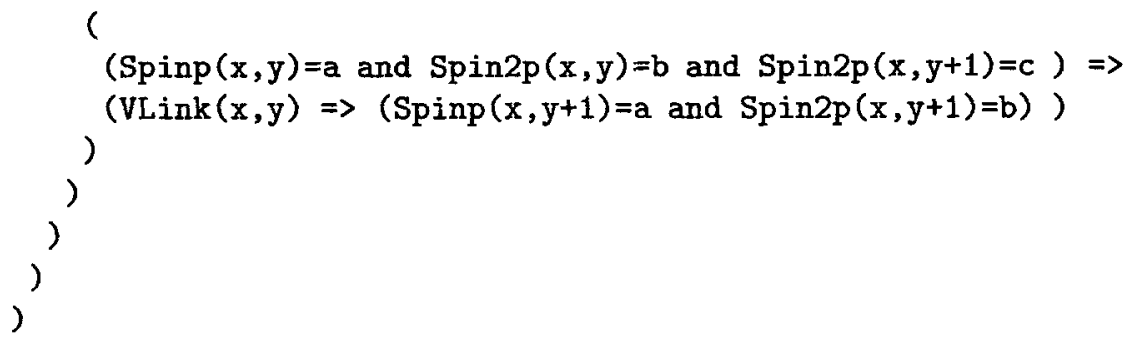

In this theorem, we start by giving values to variables $k 1$ and $k 2$. These variables define, respectively, half of the width and half of the height of the grid. With these conditions, we want to prove that after the execution of both the sequential codes (specified by the first call to the formula Algo_seq on the whole grid), and the parallel one (specified by the four last calls to the same formula with different parameters) and the glue conditions (specified by the calls of VerticalGlueing and HorizontalGlueing) we have the same graphs. The grid obtained in the sequential case is Spinp, and the grid obtained in all 
the parallel processes is Spin2p. All the sub-grids work on a different location. They all have a width equal to $\mathrm{k} 1$ and a height equal to $\mathrm{k} 2$. But they all have a different offset, respectively equal to $(0,0),(\mathrm{k} 1,0),(0, \mathrm{k} 2)$ and $(\mathrm{k} 1, \mathrm{k} 2)$. The glue postcondition modifies the value of Spin2p by assembling partial graphs linked by either a HLink if $\mathrm{x}=\mathrm{k} 1-1$ or a VLink if $\mathrm{y}=\mathrm{k} 2-1$.

Then the second part of the theorem corresponds to the equivalence between the sequential and the parallel code of each point of the grid. We prove that we obtain the same graphs in the two versions of our algorithm. We do not attempt to prove that all the points have the same value, since we do not use the same random number generator.

We have two parts corresponding respectively to the horizontal and the vertical case. In the first case, for all points $(x, y)$ of the grid, if the value of Spin, obtained after the sequential code, equals $a$, if the value of the parallel code is $b$ and the value in $(x+1, y)$ is equal to $c$ another value, then in presence of a HLink in $(x, y)$, the value of the left neighbour (in $(x+1, y)$ ) is, in the sequential case, a and, in the parallel case, $b$. The second case is considered symmetrically.

The proof of this theorem is obvious for all points not on these boundaries. For the others, we use the formula of the glue code and the proof is not difficult.

How can we verify that the specification we express corresponds to the code we abstract from? If we try to prove that the specification is correct we must prove it using known proof methods and we must prove the algorithm. In our case, we do not want to do that because we are interested in proving only that the parallelization is correct. We suppose that the sequential code is correct. Nevertheless, we can verify easily that our specification is valid during the execution of the program by injecting all formulas specified in the PVS language into the Fortran code. Of course this method can not guarantee that our specification is correct but with it we can have a good intuition of the correctness. Moreover, if we choose a too simple postcondition we could do nothing concerning the parallelization into PVS. Thus this injection of code seems us to be the appropriate way to convince ourselves of the relevance of our specification.

\section{Discovery of the glue postcondition using PVS}

We have seen that an important point of our methodology consists of proving that a parallel program is correct with respect to the sequential one from which it is developed. But our method also provides a good way of finding the glue condition to parallelize a program. For that, we define the postcondition of the sequential algorithm. Then we partition data in the Monte Carlo simulation this consists of defining sub-domains that are sub-grids where we can apply the sequential algorithm. If we try to prove that the sequential program is implied or equivalent, this depends from which point of view we take. From the parallel point of view, the prover cannot prove all the proof obligations since some data on the boundaries are not computed. But the unproven obligation proofs define the postcondition of the glue code if the specification is well designed. Unfortunately, in most cases, it is difficult to conclude that a specification is correct if we have 
not proved it. Nevertheless, with some experience, the intuition of correctness of a specification can be achieved.

We give an example with the Monte Carlo simulation glueing code search. Suppose we have no idea of the glue postcondition needed to assemble all the graphs given by the sequential algorithm applied on the sub-grids. If we try to prove the theorem EQUIVALENCE, in section 5, without the two lines VerticalGlueing and HorizontalGlueing specifying the glue code, using PVS, we obtain many unproven proof obligations. Consider the following fragment, in which we hide all the non-relevant formulas.

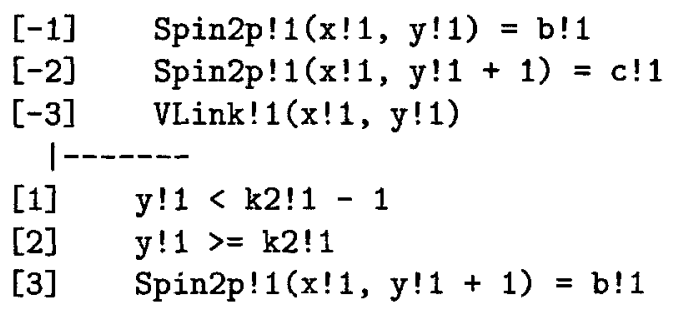

In this sequent, we have 3 formulas preceded by a negative number, these are antecedent formulas (formulas with a positive number are consequent formulas). Formulas [1] and [2] express negation of $y ! 1>=k 2 ! 1-1$ and $y ! 1<k 2 ! 1$. That is why we could obtain the following equivalent sequent in which we introduce a new formula [-1] which expresses the same fact that $\mathrm{y} ! 1>=\mathrm{k} 2 ! 1-1$ and $\mathrm{y} ! 1<\mathrm{k} 2$ ! 1 .

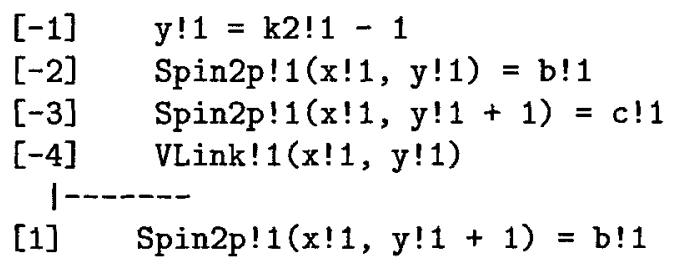

Then, if we try to compare this second sequent with the HorizontalGlueing formula, we can see the similarity between both of them. In the sequent, all variables are suffixed by $! 1$ (by PVS). If we instanciate $a$ and $b$ of HorizontalGlueing by $b$ and $c$ of the second sequent, we can conclude with this formula that $b=c$. The second sequent allows us to conclude that $b=c$ : the hypothesis of sequent and formula are equivalent. Thus, without specifying the formula HorizontalGlueing, this unproven proof obligation expresses the same fact; so we can build the postcondition expressing the horizontal glueing in PVS.

\section{Related work}

The characterization of our approach is the combination of a formal approach with practical considerations, especially the use of a theorem prover to help in the parallelization of existing codes. UNITY [2] is a way to define abstraction in 
a language of action systems; correctness properties are expressed in a fragment of the linear temporal logic. Properties are then proven using the proof system, where several systems have been developed to assist the proof of UNITY programs (see, for example, HOL-UNITY [1] and UV [7]). UNITY is oriented towards the refinement of specifications and programs and we use the refinement together with the abstraction; results from UNITY may be reused in our framework and be applied on real case studies. PCN [3] is an approach that is probably related to our experiment, since it provides a way to combine different pieces of codes with respect to combinators and under the definitional variables. In PCN, the key concept is the definitional variables used for communicating values between processes or for synchronizing concurrent activities. A PCN specification states a precondition and a postcondition over variables and provides rules for composing specifications. The strong requirement over the non-sharing of common variables between processes makes proofs easier and aids the design of code. Our experiment is another viewpoint onto the same process, and it emphasizes the process itself directly using PVS. There is no definitional variable in our approach but mathematical variables may play the role of definitional variables.

\section{Conclusion}

The use of a theorem prover, such as PVS, to parallelize a real and existing problem probably appears to be an unusual way to re-engineer existing code. The main advantage is to obtain correct code and to justify the way to compose pieces of programs. Furthermore, the decomposition was driven by the shape of the domain but parallelization patterns can be extracted from our case study and reused in other problems. The decomposition of our example was very simple but we have really developed a new parallel code for this kind of problem, in cooperation with physicists who do not take care over the correctness of programs but do require efficiency.

Another point is related to the efficiency of the produced code. First of all, the abstraction allows us to reason at the heart of the parallelization problem. Secondly, the abstraction can be used to define a measure for the complexity.

Future work will explore other case studies, and will develop a library of proved patterns for parallelizing existing codes through the abstraction mechanism and using PVS for the validation. The approach is very similar to the works of Lindsay [9], but on a class of problems for scientific applications.

\section{References}

1. F. Anderson. A theorem prover for UNITY in Higher Order Logic. $\mathrm{PhD}$ thesis, Technical University Denmark, 1992.

2. K. M. Chandy and J. Misra. Parallel Program Design A Foundation. AddisonWesley Publishing Company, 1988. ISBN 0-201-05866-9.

3. K.M. Chandy and S. Taylor. An Introduction to Parallel Programming. Jones and Bartlett, 1992. 
4. J.-P. Gibson and D. Méry. A Unifying Model for Specification and Design. In D. Galmiche J.P. Bashoun, J. Fiadeiro and A. Yonezawa, editors, Proceedings of the Workshop on Proof Theory of Concurrent Object-Oriented Programming, Linz, July 1996.

5. J.-P. Gibson and D. Méry, A unifying framework for multi-semantic software development. In Max Mühlhäuser, editor, Special Issues in Object-Oriented Programming. Dpunkt, 1997.

6. C. B. Jones and R. C. Shaw. Case Studies in Systematic Software Development. Prentice-Hall International Series in Computer Science. Prentice-Hall, 1990. ISBN0-13-116088-5.

7. M. Kaltenbach. Model checking for unity. Technical Report TR94-31, Department of Computer Sciences, The University of Texas at Austin, December 1994.

8. Leslie Lamport. A temporal logic of actions. ACM Transactions on Programming Languages and Systems, 16(3):872-923, May 1994.

9. D. Hemer P. A. Lindsay. Reuse of verified design templates through extended pattern matching. Technical report, Queensland, 1997.

10. S. Owre, N. Shankar, and J. M. Rushby. The PVS specification language. Technical report, SRI International, June 14, 19931993.

11. B. Berche P.E. Berche, C. Chatelain. Aperiodicity-induced second-order phase transition in the 8-state potts model. Physical Review Letters. To appear.

12. W. Gropp E. Lusk A. Skjellum. Using MPI : portable parallel programming with the message passing interface. MIT Press, 1994.

13. J. M. Spivey. Understanding $Z$ : a specification language and its formal semantics. Cambridge University Press, 1987.

14. A. Geist A. Beguelin J. Dongarra W. Jiang R. Manchek V. Sunderam. $P V M: A$ Users' Guide and Tutorial for Networked Parallel Computing. MIT Press, 1994.

15. I. Vattulainen. News tests of random numbers for simulations in physical systems. PhD thesis, University of Helsinki, Finland, 1994. 